\title{
Effect of lymph node dissection on stage- specific survival in patients with upper urinary tract urothelial carcinoma treated with nephroureterectomy
}

Ting-Shuai Zhai ${ }^{1+}$, Liang Jin ${ }^{1+}$, Zhen Zhou ${ }^{1,3}$, Xiang Liu ${ }^{1,4}$, Huan Liu', Wei Chen ${ }^{2}$, Jing-Yi Lu ${ }^{5}$, Xu-Dong Yao ${ }^{1 *}$, Li-Ming Feng ${ }^{2^{*}}$ and Lin Ye $\mathrm{e}^{1,5^{*}}$

\begin{abstract}
Background: We aimed to estimate the stage-specific impact of lymph node dissection (LND) on survival for upper urinary tract urothelial carcinoma (UTUC) patients treated with nephroureterectomy (NU).

Methods: Overall, 7278 UTUC patients undergoing NU within the SEER database from 2004 to 2015 were identified. Kaplan-Meier plots illustrated overall survival (OS) and cancer-specific survival (CSS) rates according to LND status. Multivariable Cox regression analyses assessed the effect of LND on OS and CSS rates stratified by pathological tumor stage.

Results: LND was performed in $26.9 \%$ of patients, and in 18.6, 23.3, 31.2 and $45.9 \%$ for pT1, pT2, pT3 and pT4 patients, respectively $(P<0.001)$. In multivariable Cox regression analyses, LND was associated with a higher OS or CSS in UTUC patients with pT3 and PT4 disease (all $P<0.05$ ), but failed to achieve independent predictor status in patients with PT1 and PT2 disease (all $P>0.05$ ). LND with 1 to 3 regional lymph nodes removed was prone to a higher OS or CSS only in PT4 compared to no LND (both $P<0.01$ ). LND with 4 or more regional lymph nodes removed predisposed to a higher OS or CSS in PT3 or PT4 (all $P<0.05$ ).
\end{abstract}

Conclusions: The beneficial effect of LND especially LND with 4 or more regional lymph nodes removed on survival was evident in pT3/4 patients. LND can be considered for $\mathrm{pT} 3$ and $\mathrm{pT} 4$, for $\mathrm{pT} 1 / 2$ remains to be seen, both of which will be verified by further prospective studies.

Keywords: Lymph node dissection, Neoplasm staging, SEER program, Survival analysis, Upper urinary tract urothelial carcinoma

\section{Background}

Upper urinary tract urothelial carcinomas (UTUCs) are rare tumors, which account for only $5-10 \%$ of urothelial carcinomas (UCs) $[1,2]$. Nephroureterectomy (NU) is the standard surgical management for high-risk UTUC [2]. At NU, lymph node dissection (LND) allows for

\footnotetext{
*Correspondence: yaoxudong67@sina.com; 2357538246@qq.com; ericyelin@163.com

${ }^{+}$Ting-Shuai Zhai and Liang Jin contributed equally to this work.

'Department of Urology, Shanghai Tenth People's Hospital of Tongji

University, School of Medicine, Shanghai 200072, China

2Department of Urology, Shawan People's Hospital, Shawan, Xinjiang 832100,

China

Full list of author information is available at the end of the article
}

optimal tumor staging and improves the detection accuracy of lymph node metastases (LNM) [3, 4]. Moreover, LND might have a potential benefit on survival outcomes for advanced-stage UTUC patients [5]. However, there are some disadvantages resulting from LND such as long surgical time and increased postoperative complications [6].

Based on the observations above, the European Association of Urology practice guidelines recommended a lymphadenectomy in patients with high-risk tumors while the strength rating was weak [2]. LND was omitted in a large amount of UTUC patients. A recent research observed that LND was performed in only $36 \%$ of UTUC

(c) The Author(s). 2019 Open Access This article is distributed under the terms of the Creative Commons Attribution 4.0 International License (http://creativecommons.org/licenses/by/4.0/), which permits unrestricted use, distribution, and 
patients who underwent NU and LND was performed more frequently in patients treated with open NU [7]. LND omission in these patients might decrease survival rate according to the literatures. To date, the curative role of LND remains debated. Few previous studies have evaluated the effect of LND on survival for UTUC patients with different tumor stage separately. To resolve this issue, we tested the impact of LND at NU on overall survival (OS) and cancer-specific survival (CSS). Our hypothesis stated that LND may benefit OS and CSS, which is consistent across all tumor stages.

\section{Methods}

\section{Study population}

Within the Surveillance, Epidemiology and End Results (SEER) database from 2004 to 2015, we identified 7278 patients with histologically confirmed UTUC who underwent NU with (1 to 3 / 4 or more regional lymph nodes removed) or without LND. Only patients with non-metastatic transitional cell carcinoma were considered. None of the patients in this study had prior history of bladder cancer. No patient with node positive disease preoperatively was included. Patients with unknown tumor stage, tumor grade and unknown LND status were excluded.

\section{Definition of variables for analyses}

Patients were stratified according to presence or absence of LND. Covariates consisted of age at diagnosis, gender (male, female), race (white, other), marital status (married, unmarried, unknown), primary site (renal pelvis, ureter), laterality (left, right, paired), tumor size $(\leq 2 \mathrm{~cm}$ and $>2 \mathrm{~cm})$, tumor stage $(\mathrm{T} 1, \mathrm{~T} 2, \mathrm{~T} 3, \mathrm{~T} 4)$, tumor grade (I, II, III, IV) and year of surgery (2004-2007, 20082011, 2012-2015).

\section{Statistical analysis}

Continuous data are reported as mean \pm s.d. and were analyzed by Student's t test. Categorical variables were compared using $X^{2}$ test or Fisher's exact test, as appropriate. Kaplan-Meier plots graphically explored overall survival (OS) and cancer-specific survival (CSS) curves. Our Cox regression analyses included three steps. Firstly, cox regression analyses tested the impact of LND (LND vs no LND) on OS and CSS. Secondly, we used cox regression analyses to estimate the relationship between LND extent and survival. Limited LND was defined as removing 1 to 3 regional lymph nodes, and extended LND was defined as removing $\geq 4$ regional lymph nodes. Thirdly, cox regression analyses were used to test the effect of lymph node stage (pN0 vs $\mathrm{pNx}$ vs $\mathrm{pN} 1-3)$ on OS and CSS. The study population was divided into lymph node-negative (N0), lymph node-positive (N1-3) (LNM), and regional lymph nodes not removed $(\mathrm{Nx})$ groups. In all multivariable analyses, covariates comprised age, gender (male vs female), race (white vs other), tumor location (renal pelvis vs ureter), laterality (left vs right), tumor size $(\leq 2 \mathrm{~cm}$ vs $>2 \mathrm{~cm}$ ), pathological tumor stage (pT1 vs pT2 vs pT3 vs pT4), lymph node stage (N0 vs N1 vs N2 vs N3) (AJCC 6th ed), tumor grade (grade I vs grade II vs grade III vs grade IV), chemotherapy (No/Unknown vs Yes) and year of surgery categories (2004-2007 vs 2008-2011 vs 2012-2015). Finally, all the aforementioned analyses were repeated for each tumor stage. The 95\% CIs were calculated and $P<0.05$ was considered statistically significant. SPSS (IBM SPSS Statistics 25) was used for analyses.

\section{Results}

\section{General characteristics}

From 2004 to 2015, 7278 patients (median age 73 years, range: 22-101) underwent NU for UTUC within the SEER database (Table 1). The majority were male (59.0\%), white race $(88.2)$, married status $(60.7 \%)$, renal pelvis $(69.1 \%)$, left Laterality (50.4\%) and big tumor size (73.6\%). Overall, 2279 patients harbored T1 (31.3\%) vs 1353 T2 (18.6\%) vs 3075 T3 (42.3\%) vs 571 T4 (7.8\%) stage and 292 patients harbored grade I (4.0\%) vs 1102 grade II (15.1\%) vs 2096 grade III (28.8\%) vs 3788 grade IV (52.0\%). Among them, $1258(17.3 \%)$ patients received adjuvant chemotherapy. 5317 (73.1\%) patients underwent NU without LND, 1961 (26.9\%) patients received LND.

\section{Trends in LND and LNM}

LND was more frequently performed in younger patients, non-white race, left laterality, bigger tumor $(>2 \mathrm{~cm})$, higher grade and advanced tumor stage (all $P<0.001$ ) (Table 1). Moreover, LND rate was increasing evidently from $2004(23.5 \%)$ to $2015(34.1 \%)(P<0.001)$ (Additional file 1: Table S1 and Figure S1, supporting information). Of the 1961 patients receiving LND, 1108 (56.5\%) were limited LND and 853 (43.5\%) were extended LND (Additional file 1: Table S2).

In patients who received LND $(n=1961)$, the overall rate of lymph node metastasis (LNM, pN1-3) was $33.9 \%$. For the same stage, LNM rates were 12.2, 20.3, 39.4 and $65.3 \%$ for pT1, pT2, pT3 and pT4 patients, respectively $(P<0.001)$. Of the 1961 patients, $56.5 \%$ underwent limited LND and $43.5 \%$ underwent extended LND, and the LNM rates were $31.4 \%$ vs $37.2 \%$, respectively $(P=0.008)$. The rate of LNM in patients who received limited LND vs extended LND was respectively 9.4 vs $15.7 \%$ for $\mathrm{pT} 1(P=0.048), 20.5$ vs $20.1 \%$ for $\mathrm{pT} 2$ ( $P=0.946), 36.3$ vs $43.6 \%$ for $\mathrm{pT} 3(P=0.022)$ and 35.1 vs $71.8 \%$ for pT4 $(P=0.058)$ (Additional file 1: Table 22 ). Extended LND was performed in respectively 44.9, 44.1, 43.1 and $42.0 \%(P=0.867)$ for patients with $\mathrm{pT} 1, \mathrm{pT} 2$, pT3 and pT4 disease, respectively. While, extended LND 
Table 1 Characteristics for UTUC patients stratified by lymph node dissection

\begin{tabular}{|c|c|c|c|c|}
\hline \multirow[t]{2}{*}{ Characteristic } & \multirow{2}{*}{$\begin{array}{l}\text { Total }^{\mathrm{a}} \\
\text { No. (\%) }\end{array}$} & \multirow{2}{*}{$\begin{array}{l}\text { No LND } \\
\text { No. }(\%)\end{array}$} & \multirow{2}{*}{$\begin{array}{l}\text { LND }^{a} \\
\text { No. (\%) }\end{array}$} & \multirow{2}{*}{$\begin{array}{l}P \\
\text { value }^{b}\end{array}$} \\
\hline & & & & \\
\hline Total & $7278(100)$ & $5317(73.1)$ & $1961(26.9)$ & \\
\hline \multicolumn{2}{|l|}{ Age (years) ${ }^{c}$} & & & $<0.001$ \\
\hline Mean \pm SD & $71.9 \pm 10.8$ & $72.4 \pm 10.8$ & $70.7 \pm 10.6$ & \\
\hline Median & 73 & 74 & 72 & \\
\hline Range & 22-101 & 22-101 & $34-96$ & \\
\hline \multicolumn{2}{|l|}{ Gender } & & & 0.904 \\
\hline Male & $4295(59.0)$ & $3140(43.1)$ & 1155 (15.9) & \\
\hline Female & $2983(41.0)$ & $2177(29.9)$ & $806(11.1)$ & \\
\hline \multicolumn{2}{|l|}{ Race } & & & $<0.001$ \\
\hline White & 6419 (88.2) & $4744(65.2)$ & 1675 (23.0) & \\
\hline Other & 859 (11.8) & $573(7.9)$ & 286 (3.9) & \\
\hline \multicolumn{2}{|l|}{ Marital status } & & & 0.284 \\
\hline Married & $4421(60.7)$ & 3205 (44.0) & $1216(16.7)$ & \\
\hline Unmarried & 2588 (35.6) & 1919 (26.4) & $669(9.2)$ & \\
\hline Unknown & $269(3.7)$ & $193(2.7)$ & $76(1.0)$ & \\
\hline \multicolumn{2}{|l|}{ Primary site } & & & 0.574 \\
\hline Renal pelvis & $5032(69.1)$ & $3686(50.6)$ & $1346(18.5)$ & \\
\hline Ureter & 2246 (30.9) & $1631(22.4)$ & $615(8.5)$ & \\
\hline \multicolumn{2}{|l|}{ Laterality } & & & $<0.001$ \\
\hline Left & 3665 (50.4) & 2576 (35.4) & 1089 (15.0) & \\
\hline Right & 3607 (49.6) & 2736 (37.6) & $871(12.0)$ & \\
\hline Paired & $6(<1 \%)$ & $5(<1 \%)$ & $1(<1 \%)$ & \\
\hline \multicolumn{2}{|l|}{ Tumor size } & & & $<0.001$ \\
\hline$\leq 2 \mathrm{~cm}$ & 1178 (16.2) & $899(12.4)$ & 279 (3.8) & \\
\hline$>2 \mathrm{~cm}$ & 5357 (73.6) & $3833(52.7)$ & 1524 (20.9) & \\
\hline Unknown & $743(10.2)$ & $585(8.0)$ & $158(2.2)$ & \\
\hline \multicolumn{2}{|l|}{ Grade } & & & $<0.001$ \\
\hline I & $292(4.0)$ & $249(3.4)$ & $43(0.6)$ & \\
\hline$\|$ & $1102(15.1)$ & $922(12.7)$ & $180(2.5)$ & \\
\hline III & 2096 (28.8) & $1492(20.5)$ & $604(8.3)$ & \\
\hline IV & 3788 (52.0) & $2654(36.5)$ & 1134 (15.6) & \\
\hline \multicolumn{2}{|l|}{ T stage } & & & $<0.001$ \\
\hline $\mathrm{T} 1$ & 2279 (31.3) & $1854(25.5)$ & $425(5.8)$ & \\
\hline $\mathrm{T} 2$ & 1353 (18.6) & 1038 (14.3) & $315(4.3)$ & \\
\hline T3 & 3075 (42.3) & $2116(29.1)$ & 959 (13.2) & \\
\hline T4 & $571(7.8)$ & $309(4.2)$ & $262(3.6)$ & \\
\hline \multicolumn{3}{|c|}{ Lymph node status status } & & - \\
\hline $\mathrm{pNx}$ & 5317 (73.1) & $5317(73.1)$ & - & \\
\hline pNO & $1296(17.8)$ & - & 1296 (17.8) & \\
\hline $\mathrm{pN} 1-3$ & $665(9.1)$ & - & $665(9.1)$ & \\
\hline \multicolumn{2}{|l|}{ Chemotherapy } & & & $<0.001$ \\
\hline No/Unknown & $6020(82.7)$ & $4635(63.7)$ & 1385 (19.0) & \\
\hline
\end{tabular}

Table 1 Characteristics for UTUC patients stratified by lymph node dissection (Continued)

\begin{tabular}{cllll}
\hline Characteristic & $\begin{array}{l}\text { Total }^{\mathrm{a}} \\
\text { No. (\%) }\end{array}$ & $\begin{array}{l}\text { No LND } \\
\text { No. (\%) }\end{array}$ & $\begin{array}{l}\text { LND }^{\mathrm{a}} \\
\text { No. (\%) }\end{array}$ & $\begin{array}{l}\text { Palue } \\
\text { Yes }\end{array}$ \\
\hline Year of surgery & & $682(9.4)$ & $576(7.9)$ & \\
2004-2007 & $2424(33.3)$ & $1865(25.6)$ & $559(7.7)$ & $<0.001$ \\
$2008-2011$ & $2451(33.7)$ & $1803(24.8)$ & $648(8.9)$ & \\
$2012-2015$ & $2403(33.0)$ & $1649(22.7)$ & $754(10.4)$ & \\
\hline
\end{tabular}

${ }^{a}$ With percentages in parentheses; ${ }^{b}$ Fisher's exact test or $x^{2}$ test, except

'Student's t test. LND, Lymph Node Dissection

was performed in 41.4, 35.8, 61.7 and $70.6 \%$ for patients with AJCC N0, N1, N2 and N3, respectively $(P<0.001)$ (Additional file 1: Table S3). The LND rates were 19.8, 86.5, 93.4 and $85.0 \%$ for AJCC N0, N1, N2 and N3 patients $(p<0.001)$ (Additional file 1: Table S4).

\section{Survival analyses according to LND status}

The 5-year and 10-year OS and CSS rates for all pT stages patients according to LND status were shown in Table 2. For LND vs no LND patients, the 5 -year OS rates and CSS rates were 41.5 vs $47.1 \%$ and 65.8 vs 74.3\%. Stage-specific 5-year OS rates for LND vs no LND patients were 66.3 vs $64.5 \%$ for pT1, 50.1 vs $47.5 \%$ for pT2, 35.3 vs $36.2 \%$ for pT3, and 13.9 vs $12.8 \%$ for pT4 disease. The 5-year CSS rates for the same tumor stages were 87.6 vs $88.9 \%, 75.6$ vs $78.6 \%, 59.2$ vs $62.4 \%$ and 31.8 vs $31.2 \%$, respectively.

In patients with limited LND vs extended LND, the 5year OS and CSS rates were 39.2 vs $44.9 \%$ and 63.5 vs 69.0\%. In patients with pT1, pT2, pT3 and pT4 disease, the 5 -year OS rates were 64.1 vs $68.9 \%$, 48.6 vs $52.0 \%$, 32.3 vs $39.6 \%$ and 15.1 vs $11.5 \%$ in patients with respectively limited LND vs extended LND. For the stage, the 5-year CSS rates were 84.7 vs $91.0 \%, 73.3$ vs $79.1 \%, 55.8$ vs $63.9 \%$ and 36.6 vs $23.8 \%$ in patients with respectively limited LND vs extended LND. In patients with pN0 vs pNx vs pN1-3 UTUC cancer, the 5-year OS and CSS rates were 52.0 vs 47.1 vs $20.7 \%$ and 76.8 vs 74.3 vs $40.1 \%$. At 5 years, the adverse impact of LND omission (pNx) and of LNM (pN1-3) compared to pN0 was consistent across all tumor stages for both OS and CSS. Kaplan-Meier plots depicting OS and CSS rates, after stratification according to LND status, LND extent and lymph node stage were shown in Fig. 1a, Fig. 1b and Fig. 1c respectively. And Kaplan-Meier plots depicting OS and CSS for stage-specific disease stratifying T stage was shown in Fig. 2. The trend of 5-year OS and CSS rates across all tumor stage according to LND status was shown in Additional file 1: Figure S2 and Figure S3.

In multivariable $\mathrm{COX}$ regression analyses, patients who underwent LND had lower hazard ratio (HR) for 
Table 2 Life of the 5-year and 10-year overall survival and cancer-specific survival rates

\begin{tabular}{|c|c|c|c|c|}
\hline \multirow{3}{*}{ All stages } & \multicolumn{2}{|c|}{ Overall survival rate (\%) } & \multicolumn{2}{|c|}{ Cancer-specific survival rate (\%) } \\
\hline & 5 years & 10 years & 5 years & 10 years \\
\hline & \multicolumn{4}{|c|}{ All stages } \\
\hline \multicolumn{5}{|l|}{ LND status } \\
\hline LND & 41.5 & 26.2 & 65.8 & 60.3 \\
\hline No LND & 47.1 & 29.2 & 74.3 & 67.8 \\
\hline \multicolumn{5}{|l|}{ LND extent } \\
\hline No LND & 47.1 & 29.2 & 74.3 & 67.8 \\
\hline Limited LND & 39.2 & 24.3 & 63.5 & 57.1 \\
\hline Extended LND & 44.9 & 28.5 & 69.0 & 65.0 \\
\hline \multicolumn{5}{|l|}{ Lymph node stage } \\
\hline pNO & 52.0 & 32.5 & 76.8 & 71.9 \\
\hline $\mathrm{pNx}$ & 47.1 & 29.2 & 74.3 & 67.8 \\
\hline $\mathrm{pN} 1-3$ & 20.7 & 13.8 & 40.1 & 34.2 \\
\hline \multicolumn{5}{|l|}{ pT1 stage } \\
\hline \multicolumn{5}{|l|}{ LND status } \\
\hline LND & 66.3 & 46.3 & 87.6 & 86.1 \\
\hline No LND & 64.5 & 41.5 & 88.9 & 83.1 \\
\hline \multicolumn{5}{|l|}{ LND extent } \\
\hline No LND & 64.5 & 41.5 & 88.9 & 83.1 \\
\hline Limited LND & 64.1 & 43.6 & 84.7 & 83.4 \\
\hline Extended LND & 68.9 & 50.9 & 91.0 & 89.1 \\
\hline \multicolumn{5}{|l|}{ Lymph node stage } \\
\hline pNO & 69.3 & 47.8 & 90.3 & 89.5 \\
\hline $\mathrm{pNx}$ & 64.5 & 41.5 & 88.9 & 83.1 \\
\hline $\mathrm{pN1} 1-3$ & 44.1 & 34.7 & 64.7 & 58.2 \\
\hline \multicolumn{5}{|l|}{ pT2 stage } \\
\hline \multicolumn{5}{|l|}{ LND status } \\
\hline LND & 50.1 & 27.3 & 75.6 & 67.3 \\
\hline No LND & 47.5 & 27.6 & 78.6 & 69.9 \\
\hline \multicolumn{5}{|l|}{ LND extent } \\
\hline No LND & 47.5 & 27.6 & 78.6 & 69.9 \\
\hline Limited LND & 48.6 & 29.7 & 73.3 & 64.0 \\
\hline Extended LND & 52.0 & 23.8 & 79.1 & 71.9 \\
\hline \multicolumn{5}{|l|}{ Lymph node stage } \\
\hline pNO & 56.5 & 29.8 & 80.6 & 72.3 \\
\hline $\mathrm{pNx}$ & 47.5 & 27.6 & 78.6 & 69.9 \\
\hline $\mathrm{pN} 1-3$ & 22.9 & 16.3 & 51.6 & 42.9 \\
\hline \multicolumn{5}{|l|}{ pT3 stage } \\
\hline \multicolumn{5}{|l|}{ LND status } \\
\hline LND & 35.3 & 22.7 & 59.2 & 52.6 \\
\hline No LND & 36.2 & 22.1 & 62.4 & 55.6 \\
\hline \multicolumn{5}{|l|}{ LND extent } \\
\hline No LND & 36.2 & 22.1 & 62.4 & 55.6 \\
\hline Limited LND & 32.3 & 19.6 & 55.8 & 47.8 \\
\hline
\end{tabular}


Table 2 Life of the 5-year and 10-year overall survival and cancer-specific survival rates (Continued)

\begin{tabular}{|c|c|c|c|c|}
\hline & \multicolumn{2}{|c|}{ Overall survival rate (\%) } & \multicolumn{2}{|c|}{ Cancer-specific survival rate (\%) } \\
\hline & 5 years & 10 years & 5 years & 10 years \\
\hline Extended LND & 39.6 & 24.5 & 63.9 & 60.4 \\
\hline \multicolumn{5}{|l|}{ Lymph node stage } \\
\hline pNO & 43.8 & 26.6 & 69.3 & 63.3 \\
\hline $\mathrm{pNx}$ & 36.2 & 22.1 & 62.4 & 55.6 \\
\hline pN1-3 & 22.2 & 14.6 & 42.1 & 35.2 \\
\hline \multicolumn{5}{|l|}{ PT4 stage } \\
\hline \multicolumn{5}{|l|}{ LND status } \\
\hline LND & 13.9 & 8.6 & 31.8 & 30.2 \\
\hline No LND & 12.8 & 4.8 & 31.2 & 28.8 \\
\hline \multicolumn{5}{|l|}{ LND extent } \\
\hline No LND & 12.8 & 4.8 & 31.2 & 28.8 \\
\hline Limited LND & 15.1 & 8.3 & 36.6 & NA \\
\hline Extended LND & 11.5 & 9.2 & 23.8 & NA \\
\hline \multicolumn{5}{|l|}{ Lymph node stage } \\
\hline pNO & 21.6 & 14.8 & 48.1 & NA \\
\hline $\mathrm{pNx}$ & 12.8 & 4.8 & 31.2 & 28.8 \\
\hline pN1-3 & 9.9 & 4.5 & 19.6 & NA \\
\hline
\end{tabular}

OS (HR 0.87, $P<0.01$ ) and CSS (HR 0.81, $P<0.01$ ) rates compared to patients operated without LND (Tables 3 and 4). When stratifying according to tumor stage, the beneficial impact of LND on OS remained consistent for patients with pT3 or pT4 disease while disappeared for patients with $\mathrm{pT} 1$ or $\mathrm{pT} 2$ disease. Patients receiving extended LND (HR 0.83, $P<0.01$ ) or limited LND (HR $0.90, P<0.05)$ had evidently higher OS rate compared to no LND (Reference category). After the analyses were repeated across all pathological stages, the beneficial impact of extended LND on OS was only found in pT3 or pT4 stages (HR 0.80, $P<0.05$, HR $0.77, P<0.05$ respectively). While, the protective effect of limited LND was only observed in patients with pT4 stage and its beneficial role seemed to be more evident than extended LND (limited LND vs extended LND: HR 0.73, $P<0.01$ vs HR $0.77, P<0.05)$. Similar results were found when CSS rates were tested (Tables 3 and 4). Finally, the OS rate was 1.2 -fold and 2.2-fold higher (both $P<0.001$ ) in $\mathrm{pN} 0$ patients relative to those with $\mathrm{pNx}$ and $\mathrm{pN} 1-3$ disease respectively (Tables 3 and 4), which were consistent when same analyses were performed across all tumor stages. Similar results were produced when CSS rates were tested.

\section{Discussion}

Our study aimed to determine the impact of LND on survival in UTUC patients following NU. In this study, LND was performed in only $26.9 \%$ of the UTUC patients, which seemed to be infrequent compared to the
LND rate $75.0 \%$ at radical cystectomy (RC) for nonmetastatic bladder transitional cell carcinoma [8]. While, LND was increasingly considered at NU year by year from 2004 (23.5\%) to 2015 (34.1\%), which indicated that urologists were increasingly aware of the crucial role of LND. And this trend was consistent with a previous UTUC study performed by Chappidi et al. [9]. Moreover, younger patients, non-white race, left laterality, bigger tumor $(>2 \mathrm{~cm})$, higher grade and advanced tumor stage were important factors contributing to the decisionmaking of LND. Kaplan-Meier plots illustrated that extended LND with 4 or more regional lymph nodes removed might benefit OS or CSS for pT1-pT3 and limited LND with 1 to 3 regional lymph nodes removed may benefit OS or CSS for pT4 disease, although not to a significant extent (Fig. 2, Additional file 1: Figure S2 and Figure S3). However, in multivariable Cox regression analyses, LND was beneficial for UTUC patients especially for pT3 and pT4 disease. Extended LND brought evident benefits to $\mathrm{pT} 3$ and pT4 patients, while limited LND was only beneficial for pT4 patients and more effective than extended LND. These findings suggested LND could be considered at NU for $\mathrm{pT} 3 / 4$, for pT1/2 remains to be seen, both of which would be verified by further prospective studies.

At present, the therapeutic role of LND at NU for UTUC patients remains controversial [10-13]. Most studies worked on the effect of LND on survival in all stage patients. However, LND was seldom considered unless preoperative examinations indicated a high 

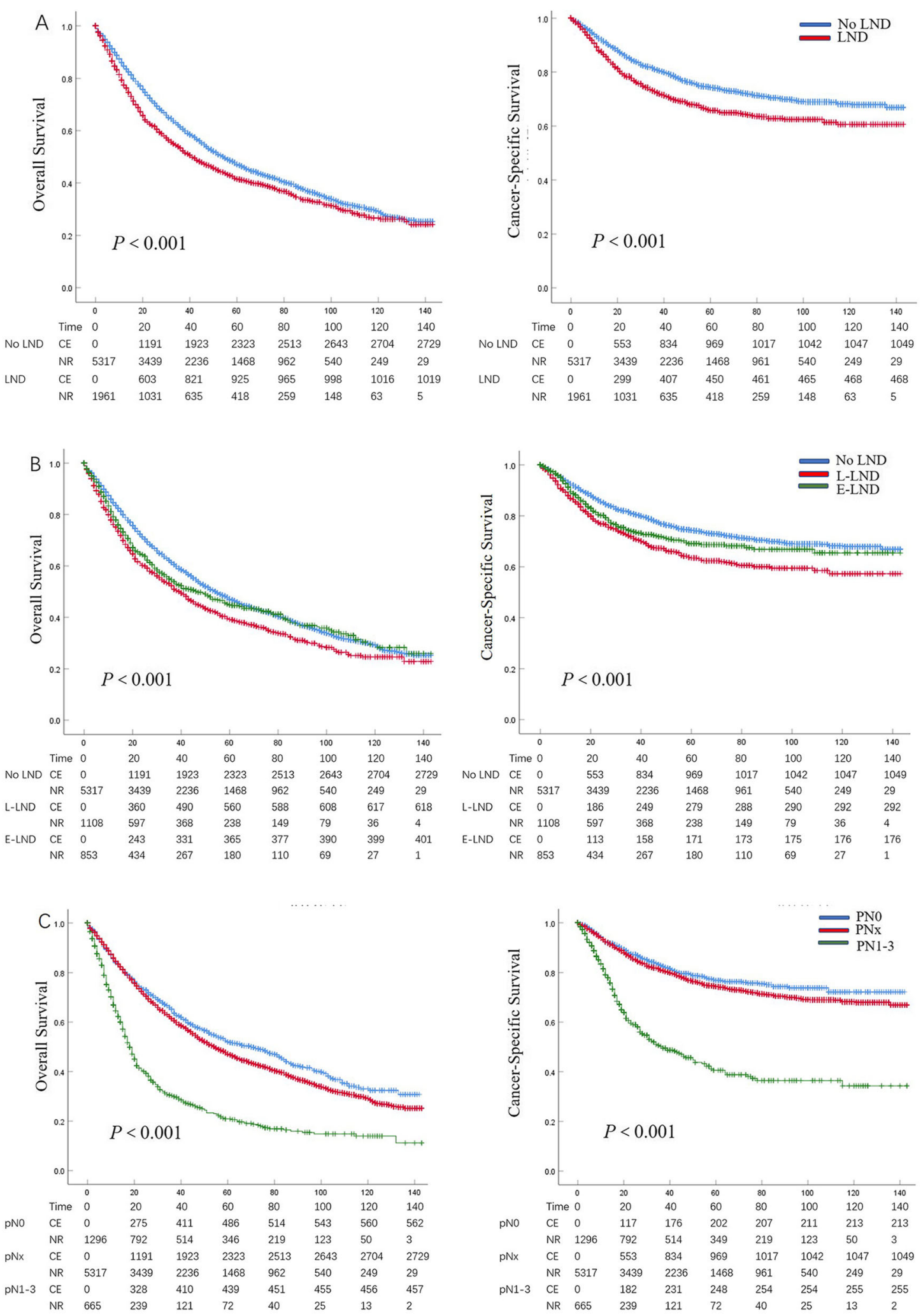

Fig. 1 (See legend on next page.) 
(See figure on previous page.)

Fig. 1 Kaplan-Meier plots depicting overall survival (OS) and cancer-specific survival (CSS), after stratification according to lymph node dissection (LND) status (a), LND extent (b), and lymph node stage (c) in 7278 patients treated with radical cystectomy between 2004 and 2015 , within the Surveillance, Epidemiology and End Results database. C. E, cumulative number of events; N. R, number at risk; L-LND, Limited LND; E-LND, Extended LND

probability of tumor invasiveness or LNM. Therefore, the patients undergoing LND were prone to worse pathologic tumor stage compared to those without LND, which resulted in the bias for survival analysis. As a result, we performed this stage-specific study to eliminate the bias and investigate whether LND could bring benefits to survival. Also, we included the extent of LND in the analysis since previous studies had stressed its clinical role $[8,9,14]$.

Firstly, the LND rate was evidently increasing from 2004 to 2015 (Additional file 1: Figure S1), which indicated that urologists were likely to perform LND at NU for UTUC patients. A recent study performed by Chappidi et al. [9] described a similar increasing trend from $20 \%$ in 2004 to $33 \%$ in 2012 for UTUC patients. Similar trends were also found in other cancers $[8,15]$, which suggested LND was becoming more and more important in cancer therapy. Secondly, LND were likely to be performed in younger, non-white race, left laterality, bigger tumor $(>2 \mathrm{~cm})$, higher grade and advanced tumor stage patients according to this study, which may due to the good surgical tolerance of young patients and higher probability of aggressive tumor in these patients. Then, we found that LND might improve OS and CSS across all tumor stages especially for pT3 to pT4 disease from Kaplan-Meier plots and COX regression analysis. To further investigate the effect of LND extent on the prognosis, we divided the LND population into two groups: limited LND and extended LND. The plots described patients who underwent LND with 4 or more regional lymph node removed survived longer than those in pT1 to pT3 stages. While, patients receiving LND with 1 to 3 regional lymph nodes removed survived the longest in pT4 stage. In COX analysis, extended LND improved survival significantly in pT3 to pT4 disease, while limited LND only improved the survival of pT4 patients evidently and it seemed more beneficial than extended LND. This may due to the higher incidence of severe complications resulting from extended LND in pT4. However, this may also due to selection bias. Anyway, it needs to be verified by further prospective studies. In the past few years, there were studies working on the effect of LND for UTUC patients. Sebastiano Nazzani et al. reported that LND could provide extensive prognostic information [16]. According to Fan Dong et al. [13], LND could improve survival for clinically node-

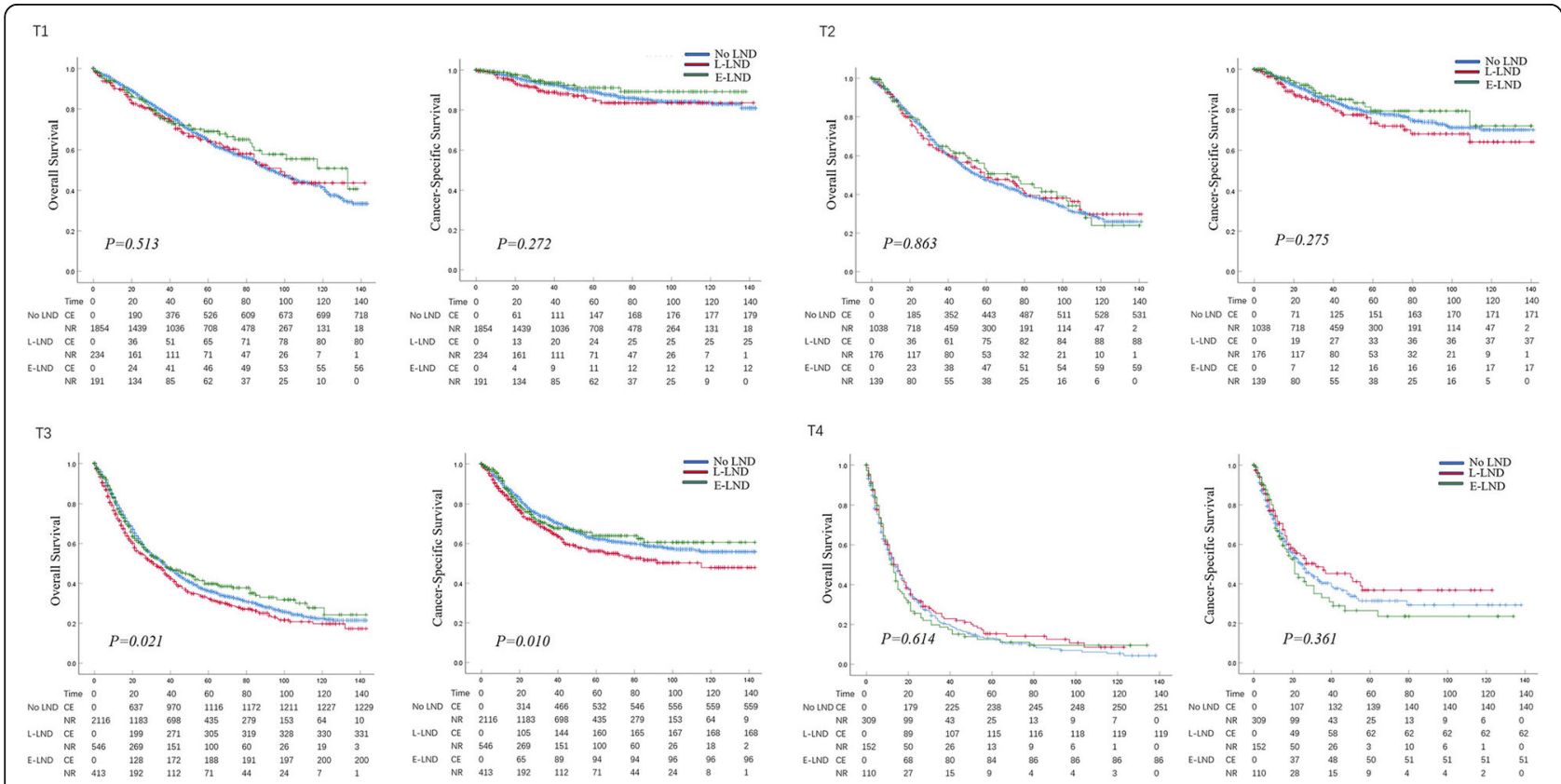

Fig. 2 Kaplan-Meier plots depicting overall survival (OS) and cancer-specific survival (CSS) for pT1, pT2, pT3 and pT4 diseases, after stratification according to LND extent in 7278 patients treated with radical cystectomy between 2004 and 2015, within the Surveillance, Epidemiology and End Results database. C. E, cumulative number of events; N. R, number at risk; L-LND, Limited LND; E-LND, Extended LND 
Table 3 Multivariable Cox regression analyses predicting overall survival

\begin{tabular}{|c|c|c|c|c|c|}
\hline & All stages & $\mathrm{T} 1$ & $\mathrm{~T} 2$ & $\mathrm{~T} 3$ & T4 \\
\hline & $\begin{array}{l}\text { Multivariable }{ }^{a} \\
\text { HR }(95 \% \mathrm{Cl})\end{array}$ & $\begin{array}{l}\text { Multivariable b } \\
\text { HR }(95 \% \mathrm{Cl})\end{array}$ & $\begin{array}{l}\text { Multivariable b } \\
\text { HR }(95 \% \mathrm{Cl})\end{array}$ & $\begin{array}{l}\text { Multivariable b } \\
\text { HR }(95 \% \mathrm{Cl})\end{array}$ & $\begin{array}{l}\text { Multivariable b } \\
\text { HR }(95 \% \mathrm{Cl})\end{array}$ \\
\hline \multicolumn{6}{|l|}{ LND status } \\
\hline No LND & 1.00 (Ref.) & 1.00 (Ref.) & 1.00 (Ref.) & 1.00 (Ref.) & 1.00 (Ref.) \\
\hline LND & $0.87(0.80-0.95)^{* *}$ & $0.98(0.80-1.19)$ & $0.85(0.70-1.04)$ & $0.88(0.78-0.99)^{*}$ & $0.74(0.59-0.94)^{*}$ \\
\hline \multicolumn{6}{|l|}{ LND extent } \\
\hline No LND & 1.00 (Ref.) & 1.00 (Ref.) & 1.00 (Ref.) & 1.00 (Ref.) & 1.00 (Ref.) \\
\hline Limited LND & $0.90(0.81-0.99)^{*}$ & $0.98(0.78-1.25)$ & $0.87(0.69-1.11)$ & $0.93(0.81-1.07)$ & $0.73(0.57-0.94)^{* *}$ \\
\hline Extended LND & $0.83(0.74-0.94)^{* *}$ & $0.97(0.73-1.30)$ & $0.82(0.61-1.09)$ & $0.80(0.68-0.95)^{*}$ & $0.77(0.57-0.99)^{*}$ \\
\hline \multicolumn{6}{|l|}{ Lymph node stage } \\
\hline pNO & 1.00 (Ref.) & 1.00 (Ref.) & 1.00 (Ref.) & 1.00 (Ref.) & 1.00 (Ref.) \\
\hline $\mathrm{pNx}$ & $1.19(1.08-1.30)^{* * *}$ & $1.05(0.86-1.29)$ & $1.20(0.98-1.48)$ & $1.15(1.01-1.31)^{*}$ & $1.59(1.20-2.11)^{* *}$ \\
\hline pN1-3 & $2.20(1.93-2.50)^{* * * *}$ & $2.13(1.33-3.41)^{* *}$ & $2.33(1.60-3.40)^{* * *}$ & $2.00(1.68-2.39)^{* * *}$ & $2.26(1.65-3.10)^{* * *}$ \\
\hline
\end{tabular}

${ }^{*} P<0.05,{ }^{* *} P<0.01,{ }^{* * *} P<0.001 .{ }^{a}$ Adjusted to age, gender, race, tumor location, laterality, tumor size, tumor stage, tumor grade, chemotherapy and year of surgery. ${ }^{\mathrm{b}}$ Adjusted to age, gender, race, tumor location, laterality, tumor size, tumor grade, chemotherapy and year of surgery. $H R$ hazard ratio, $95 \%$ Cl $95 \%$ confidence interval, LND lymph node dissection

negative UTUC patients, and its protective role seemed to be more evident in muscle-invasive diseases. While, the study conducted by Inokuchi Junichi et al. indicated that there was no therapeutic benefit of LND for UTUC patients treated with NU [12]. Inokuxhi Junichi et al. did not stratify the patients according to tumor stage, thus patients with LND were more likely to suffer clinically node-positive or muscle-invasive diseases, which might result in the bias. In our study, LNM rates were 12.2, 20.3, 39.4 and $65.3 \%$ for pT1, pT2, pT3 and pT4 patients and $19.8,86.5,93.4$ and $85.0 \%$ for N0, N1, N2 and N3 patients respectively, which indicated that the stages of patients with LND were more advanced. Zareba Piotr et al. showed that a higher LN yield was associated with lower all-cause mortality [17]. Winer AG et al. consistently reported that extended LND might have a beneficial effect on oncologic outcomes in high-risk UTUC patients without increasing surgical time or risk of complications [18]. Consequently, LND especially extended LND at NU should particularly be contemplated in locally advanced UTUC patients, as the protective effect of LND on OS or CSS is more evident in these patients compared to organ-confined diseases. Furthermore, we observed that LND might have a minimal positive impact on OS or CSS in pT1/2 patients. This may result from the difficulty to standardize indication or template

Table 4 Multivariable Cox regression analyses predicting cancer-specific survival

\begin{tabular}{|c|c|c|c|c|c|}
\hline & All stage & $\mathrm{T} 1$ & $\mathrm{~T} 2$ & T3 & T4 \\
\hline & $\begin{array}{l}\text { Multivariable }{ }^{a} \\
\text { HR (95\% Cl) }\end{array}$ & $\begin{array}{l}\text { Multivariable b } \\
\text { HR }(95 \% \mathrm{Cl})\end{array}$ & $\begin{array}{l}\text { Multivariable }{ }^{\text {b }} \text { HR (95\% Cl) }\end{array}$ & $\begin{array}{l}\text { Multivariable b } \\
\text { HR (95\% CI) }\end{array}$ & $\begin{array}{l}\text { Multivariable b } \\
\text { HR }(95 \% \mathrm{Cl})\end{array}$ \\
\hline \multicolumn{6}{|l|}{ LND status } \\
\hline No LND & 1.00 (Ref.) & 1.00 (Ref.) & 1.00 (Ref.) & 1.00 (Ref.) & 1.00 (Ref.) \\
\hline LND & $0.81(0.73-0.95)^{* *}$ & $0.84(0.56-1.25)$ & $0.87(0.61-1.24)$ & $0.83(0.73-0.98)^{*}$ & $0.64(0.47-0.88)^{* *}$ \\
\hline \multicolumn{6}{|l|}{ LND extent } \\
\hline No LND & 1.00 (Ref.) & 1.00 (Ref.) & 1.00 (Ref.) & 1.00 (Ref.) & 1.00 (Ref.) \\
\hline Limited LND & $0.86(0.75-0.96)^{*}$ & $1.02(0.65-1.59)$ & $1.02(0.69-1.51)$ & $0.92(0.78-1.16)$ & $0.61(0.43-0.87)^{* *}$ \\
\hline Extended LND & $0.73(0.61-0.88)^{* *}$ & $0.56(0.29-1.08)$ & $0.65(0.38-1.12)$ & $0.74(0.58-0.95)^{*}$ & $0.70(0.47-0.96)^{*}$ \\
\hline \multicolumn{6}{|l|}{ Lymph node stage } \\
\hline pNO & 1.00 (Ref.) & 1.00 (Ref.) & 1.00 (Ref.) & 1.00 (Ref.) & 1.00 (Ref.) \\
\hline $\mathrm{pNx}$ & $1.32(1.13-1.53)^{* * * *}$ & $1.29(0.85-1.98)$ & $1.19(0.83-1.72)$ & $1.22(1.01-1.49)^{*}$ & $1.97(1.31-2.97)^{* *}$ \\
\hline $\mathrm{pN} 1-3$ & $2.49(2.06-3.01)^{* * *}$ & $3.40(1.61-7.18)^{* *}$ & $3.35(1.87-5.99)^{* * *}$ & $2.32(1.81-2.98)^{* * *}$ & $2.55(1.64-3.98)^{* * *}$ \\
\hline
\end{tabular}

${ }^{*} P<0.05,{ }^{* *} P<0.01,{ }^{* * *} P<0.001$. ${ }^{a}$ Adjusted to age, gender, race, tumor location, laterality, tumor size, tumor stage, tumor grade, chemotherapy and year of surgery. ${ }^{\mathrm{b}}$ Adjusted to age, gender, race, tumor location, laterality, tumor size, tumor grade, chemotherapy and year of surgery. $H R$ hazard ratio, $95 \%$ Cl $95 \%$ confidence interval, LND lymph node dissection 
for LND $[4,19]$. In consequence, it simply cannot be argued that LND is of no benefit for organ-confined UTUC patients.

Additionally, our results validated the detrimental impact of LNM across all tumor stages. Patients who did not receive $\mathrm{LND}(\mathrm{pNx})$ fared worse than those with absence of LNM confirmed by LND (pN0). Patients with LNM (pN1-3) validated by LND fared the worst. And these results were consistent across all stages (Tables 3 and 4). Similar studies have also reported that patients with $\mathrm{pNx}$ might have a worse prognosis compared to those with pNO [20, 21]. Therefore, LND should be recommended as long as it can be safely performed.

Several limitations of the study should be acknowledged. This study was performed in an observational manner. And the groups might differ in recorded or unrecorded variables, which may influence survival, since patients were not randomized to receive LND. Moreover, the anatomical extent of LND could not be defined since the extent of LND lacks standardization, and the number of regional lymph nodes removed was used instead as a proxy. Different treatment groups, surgeon experience, approach to radical nephroureterectomy and academic vs non-academic facility might also result in selection bias.

\section{Conclusions}

LND was more frequently performed in locally advanced UTUC patients. The beneficial effect of LND especially LND with 4 or more regional lymph nodes removed on survival was evident in pT3/4 disease, while pT1 and pT2 fell out in terms of benefit of LND. LND with 1 to 3 regional lymph nodes removed seemed to be more beneficial than LND with 4 or more regional lymph nodes removed in pT4 disease. LND can be considered for pT3 and pT4, for pT1/2 remains to be seen, both of which will be verified by further prospective studies.

\section{Supplementary information}

Supplementary information accompanies this paper at https://doi.org/10. 1186/s12885-019-6364-z.

Additional file 1: Table S1. Year of diagnosis for UTUC patients stratified by lymph node dissection. Table S2. Descriptive characteristics of 1961 LND patients according to T stage. Table S3. Extent of LND in the same stage disease. Table S4. AJCC N stage distribution according to LND status. Figure S1. Trend of LND rate from 2004 to 2015. Figure S2. Trend of 5-year OS rate across all tumor stages according to LND status. Figure S3. Trend of 5-year CSS rate across all tumor stages according to LND status.

\section{Abbreviations}

CSS: Cancer-specific survival; LND: Lymph node dissection; LNM: Lymph node metastasis; NU: Nephroureterectomy; OS: Overall survival; SEER: Surveillance, epidemiology and end results database; UTUC: Upper urinary tract urothelial carcinoma

\section{Acknowledgements}

We kindly thank the editor and reviewers for careful review and valuable comments, which have led to a significant improvement of this manuscript. We thank the Surveillance, Epidemiology and End Results (SEER) database. Professor Lin Ye financially supported the conduct of this clinical study and the publication.

\section{Authors' contributions}

TS Z contributed to conceptualization, data curation, formal analysis, investigation, methodology, validation, visualization and writing-original draft. L J contributed to data curation, formal analysis, investigation, methodology, software, validation and visualization. Z Z contributed to data curation, formal analysis, investigation, methodology and visualization. X L contributed to formal analysis, methodology and validation. $\mathrm{H} L$ contributed to data curation, formal analysis and methodology. W C contributed to supervision and writing-review and editing. JY $L$ contributed to writing-review and editing. $X D Y$ contributed to conceptualization and supervision. LM F contributed to conceptualization, supervision and writing-review and editing. L Y contributed to conceptualization, funding acquisition, resources, supervision and writing-review and editing. All authors read and approved the final manuscript.

\section{Funding}

This work was supported by grants from the National Natural Science Foundation of China (No. 81672549 and No. 81972409), Natural Science Foundation of Shanghai (No. 15ZR1433000) and Karamay Municipal Science and Technology Foundation (No. JK2017-5). The funding bodies had no role in the design of the study and collection, analysis, and interpretation of data and in writing the manuscript.

\section{Availability of data and materials}

The datasets used and/or analyzed during this current study are available from the corresponding author on reasonable request.

\section{Ethics approval and consent to participate}

For the institutional cohorts, data were extracted from the Surveillance, Epidemiology, and End Results database. This article does not contain any studies with human participants performed by any of the authors. For this type of study, formal consent is not required.

\section{Consent for publication}

Not applicable.

\section{Competing interests}

The authors declare that they have no competing interests.

\section{Author details}

${ }^{1}$ Department of Urology, Shanghai Tenth People's Hospital of Tongji University, School of Medicine, Shanghai 200072, China. ${ }^{2}$ Department of Urology, Shawan People's Hospital, Shawan, Xinjiang 832100, China. ${ }^{3}$ Nanjing Medical University, Nanjing 210000, China. ${ }^{4}$ Department of Urology, Shanghai Putuo District People's Hospital of Tongji University, School of Medicine, Shanghai 200333, China. ${ }^{5}$ Department of Urology, Karamay Central Hospital, Karamay, Xinjiang 834000, China.

Received: 3 June 2019 Accepted: 15 November 2019

Published online: 12 December 2019

References

1. Siegel RL, Miller KD, Jemal A. Cancer statistics, 2018. CA Cancer J Clin. 2018; 68:7-30.

2. Morgan Rouprêt, Marko Babjuk, Eva Compérat, Richard Zigeuner, Richard J. Sylvester, Maximilian Burger, et al. European Association Of Urology guidelines on upper urinary tract urothelial carcinoma: 2017 update. Eur Urol, 2018, 73: 111-122.

3. Roscigno $M$, Brausi $M$, Heidenreich $A$, et al. Lymphadenectomy at the time of nephroureterectomy for upper tract urothelial cancer. Eur Urol. 2011; 60(4):776-83.

4. Lughezzani $G$, Jeldres $C$, Isbarn $H$, et al. A critical appraisal of the value of lymph node dissection at nephroureterectomy for upper tract urothelial carcinoma. Urology. 2010;75(1):118-24. 
5. Dominguez-Escrig JL, Peyronnet B, Seisen T, et al. Potential benefit of lymph node dissection during radical Nephroureterectomy for upper tract Urothelial carcinoma: a systematic review by the European Association of Urology guidelines panel on non-muscle-invasive bladder cancer. Eur Urol Focus. 2017;17(2):e1721.

6. Thurairaja R, Studer UE, Burkhard FC. Indications, extent, and benefits of pelvic lymph node dissection for patients with bladder and prostate cancer. Oncologist. 2009;14:40-51.

7. Moschini M, Foerster B, Abufaraj M, et al. Trends of lymphadenectomy in upper tract urothelial carcinoma (UTUC) patients treated with radical nephroureterectomy. World J Urol. 2017;35(10):1541-7.

8. Abdollah F, Sun M, Schmitges J, et al. Stage-specific impact of pelvic lymph node dissection on survival in patients with non-metastatic bladder cancer treated with radical cystectomy. BJU Int. 2012;109(8):1147-54.

9. Chappidi MR, Kates M, Johnson MH, et al. Lymph node yield and tumor location in patients with upper tract urothelial carcinoma undergoing nephroureterectomy affects survival: A U.S. population-based analysis (2004-2012). Urol Oncol. 2016;34(12):531.e15-24.

10. Junichi I, Masatoshi E, Tomohiko H, et al. Impact of lymph node dissection on clinical outcomes during nephroureterectomy in patients with clinically node-negative upper urinary tract urothelial cancer: subanalysis of a multiinstitutional nationwide case series of the Japanese Urological Association. Jpn J Clin Oncol. 2017:47:652-9.

11. Masaomi I, Kazumasa M, Kazushige S, et al. Effect of lymphadenectomy during radical nephroureterectomy in locally advanced upper tract urothelial carcinoma. Clin Genitourin Cancer. 2017;15:556-62.

12. Junichi I, Kentaro K, Yoshiyuki K, et al. Role of lymph node dissection during radical nephroureterectomy for upper urinary tract urothelial cancer: multiinstitutional large retrospective study JCOG1110A. World J Urol. 2017;35: 1737-44.

13. Dong $F, X u T$, Wang $X$, et al. Lymph node dissection could bring survival benefits to patients diagnosed with clinically node-negative upper urinary tract urothelial cancer: a population-based, propensity score-matched study. Int J Clin Oncol. 2019;24:296-305.

14. Gschwend Jürgen $\mathrm{E}$, Heck Matthias $\mathrm{M}$, Jan $\mathrm{L}$, et al. Extended versus limited lymph node dissection in bladder cancer patients undergoing radical cystectomy: survival results from a prospective, randomized trial. Eur Urol. 2019;75:604-11.

15. Luigi N, Akshay S, Deepansh D, et al. Rate and extent of pelvic lymph node dissection in the US prostate cancer patients treated with radical prostatectomy. Clin Genitourin Cancer. 2018;16:e451-67.

16. Sebastiano N, Elio M, Felix P, et al. Rates of lymph node invasion and their impact on cancer specific mortality in upper urinary tract urothelial carcinoma. Eur I Surg Oncol. 2019;45:1238-45.

17. Piotr Z, Barak R, Winer Andrew G, et al. Association between lymph node yield and survival among patients undergoing radical nephroureterectomy for urothelial carcinoma of the upper tract. Cancer. 2017;123:1741-50.

18. Winer Andrew G, Vertosick EA, Ghanaat M, et al. Prognostic value of lymph node yield during nephroureterectomy for upper tract urothelial carcinoma. Urol Oncol. 2017:35:151.e9-151.e15.

19. Jamie M, Kuan LY, Raman Jay D. The role of lymphadenectomy for upper tract urothelial carcinoma. Nat Rev Urol. 2011;8:394-401.

20. Maximilian B, Shariat Shahrokh F, Hans-Martin F, et al. No overt influence of lymphadenectomy on cancer-specific survival in organ-confined versus locally advanced upper urinary tract urothelial carcinoma undergoing radical nephroureterectomy: a retrospective international, multi-institutional study. World J Urol. 2011;29:465-72

21. Thomas S, Shariat Shahrokh F, Olivier C, et al. Contemporary role of lymph node dissection at the time of radical nephroureterectomy for upper tract urothelial carcinoma. World J Urol. 2017;35:535-48.

\section{Publisher's Note}

Springer Nature remains neutral with regard to jurisdictional claims in published maps and institutional affiliations.

Ready to submit your research? Choose BMC and benefit from:

- fast, convenient online submission

- thorough peer review by experienced researchers in your field

- rapid publication on acceptance

- support for research data, including large and complex data types

- gold Open Access which fosters wider collaboration and increased citations

- maximum visibility for your research: over $100 \mathrm{M}$ website views per year

At BMC, research is always in progress.

Learn more biomedcentral.com/submissions 